\title{
DESCRIÇÃO DA COLONIZAÇÃO DE AEDES ALBOPICTUS (DIPTERA: CULICIDAE) NA REGLÃO DE SÃO JOSÉ DO RIO PRETO, SP, 1991-1994
}

\author{
Francisco Chiaravalloti Neto, Antonio I.P. da Costa, Margareth R. D. \\ Soares, Sirle A. S. Scandar e Rubens P. Cardoso Junior
}

\begin{abstract}
O objetizo deste trabalbo é descrever e analisar colonização do Aedes albopictus, cuja presença foi detectada na região de São José do Rio Preto em 1991, já colonizada pelo Aedes aegypti. A partir de informaçóes obtidas em medidas de densidade larvária pela Superintendência de Controle de Endemias (SUCEN), analisou-se: ano e mês de ocorrência, nunicípio, composição e localização das amostras lanárias, tipo de recipiente, número médio de lanas e Índice de Breteau. Até dezembro de 1994 a presença do Ae. albopictus fora constatada en 34 municípios. A colonização da região pelo mosquito ainda é reduzida, apresentando algumas diferenças em relação ao Ae. aegypti: maior proporção no peridonicilio, ocupando recipientes em proporçōes diferentes. O número médio de lanas de Ae. albopictus sofreu influência da presença de lartas da outra espécie. Apresentou compontamento sazonal semelhante ao do Ae. aegypti e avançou no sentido leste para oeste.
\end{abstract}

Palan ras-chares: Aedes. Aedes albopictus. Colonização. Ecologia.

Em junho de 1986 foi detectado pela primeira vez no Brasil o mosquito Aedes albopictus, no Rio de Janeiro ${ }^{\circ}$ enquanto a recolonizacão do Aedes aegypti ocorreu a partir de $1976^{\circ}$. Segundo Nobre e $\mathrm{Col}^{9}$, o Ae. albopictus já se đisseminou pelos estados de Espírito Santo, Maranhão, Minas Gerais, Paraná, Rio de Janeiro, Rondônia e São Paulo.

Um levantamento realizado em 1985 pela Superintendência de Controle de Endemias (SUCEN), órgão viniculado à Secretaria Estadual de Saúde, detectou focos de Ae. aegypti em 12 municípios do Estado de São Paulo ${ }^{12}$. No final de 1985, já existiam nove municípios com infestação domiciliar pelo Ae aegypti, todos no oeste do Estado de São Paulo'? ${ }^{12}$ Em 1986, foi encontrado foco do Ae. albopictus na região leste do estado, no Vale do Paraíba ${ }^{3}$, sendo que em 1987 foi identificada a infestação domiciliar pelo mosquito em municípios dessa região. A partir de 1985, o Ae. aegypti foi se dispersando no sentido oeste para leste e o Ae. albopictus, a partir de 1987 no sentido leste para oeste 2 .

Superintendência de Controle de Endemias (SUCEN), São José do Rio Preto, SP.

Endereşo para correspondência: Francisco Chiaravalloti Neto. Av. Philadelpho Manoel Gouveia Neto 3101 - $3^{\circ}$ ndar, Bairro Maceno 15060-040 São José do Rio Preto, SP. Recebido para publicação em 18/11/95.
Desde entâo a colonização do Estado por estas duas espécies vem se ampliando rapidamente, sendo que em dezembro de 1994, do total de 626 municipios, 406 estavam colonizados pelo Ae. aegypti e 421 pelo Ae. albopictus. Em 242 municípios, ocorria a infestação simultânea pelas duas espécies (SUCEN, dados não publicados).

$\mathrm{Na}$ região de São José do Rio Preto, localizada a oeste do Estado de São Paulo, em levantamentos realizados pela SUCEN em 1985 , foi detectada infestação domiciliar pelo $A e$. aegypti no município de São José do Rio Preto, cidade sede da região ${ }^{11}$. A infestação domiciliar do mosquito evoluiu rapidamente sendo que em maio de 1989, todos os 92 municípios da região já se encontravam colonizados domiciliarmente pelo Ae. aegypti (SUCEN, dados não publicados).

Os primeiros encontros de Ae. albopictus, na região de São José do Rio Preto, ocorreram nos municípios de Santa Adélia, Novo Horizonte e Eliziário em maio de 1991, quando da realização de atividade de medida de densidade larvária ${ }^{13}$. Verifica-se uma colonização recente, cuja descrição e análise são os objetivos deste trabalho.

\section{MATERIAL E MÉTODOS}

A SUCEN tem realizado desde 1985 medidas mensais do Índice de Breteau por 
Chiaravalloti Neto F, Costa AIP, Soares MRD, Scandlar SAS, Cardoso Jr RP. Descrição da colonização de Aedes albopictus (Diptera: Culicidae) na região de São José do Rio Preto, SP, 1991-1994. Rezista da Sociedade Brasileira de Medicina Tropical 29:543-548, nov-dez 1996.

amostragem de quadras e residências ${ }^{1}$, em todos os municípios da regiâo. Em cada medida os funcionários da SUCEN visitaram as casas das quadras sorteadas, pesquisaram recipientes existentes e coletaram amostras daqueles que continham larvas de mosquitos. As amostras foram encaminhadas aos laboratórios da SUCEN para identificação, sendo consideradas positivas aquelas contendo larvas de Ae aegypti e/ou Ae. albopictus, correspondendo cada uma também a um recipiente positivo.

Todas informações foram registradas em Boletins de Densidade Larvária utilizados pela SUCEN. A partir destes dados obteve-se:

$$
\begin{aligned}
& \text { a ocupação, mês a mês, da região pelo Ae. } \\
& \text { albopictus; }
\end{aligned}
$$

a composição das amostras positivas quanto à presença de larvas das espécies vetoras de dengue e febre amarela (somente Ae aegypti, somente Ae. albopictus ou ambas espécies);
- a localizaçào das amostras positivas nos imóveis (no intradomicílio ou no peridomićlio);

- a caracterização dos tipos de recipientes;

- os números médios de larvas encontradas por espécie e por tipo de recipiente.

Para os municípios em que foram encontradas larvas de Ae, albopictus, foram calculados os Índices de Breteau para as duas espécies vetoras, dividindo-se o número de recipientes positivos para determinada espécie pelo número de casas pesquisadas, com a multiplicação do resultado por $100^{4}$. Os Índices de Breteau por espécie foram agrupados mensalmente para cálculo das médias mensais.

Para verificação da existência de associação entre composição e localização das amostras e entre composição das amostras e tipos de recipientes ocupados. foi utilizado o teste estatístico do Qui-quadrado. Os números médios de larvas por espécie foram comparados com a utilização do teste "t de Student"

\section{RESULTADOS}

Na Tabela 1 são apresentados os números de amostras com larvas de Ae. albopictus nas sub-regiões de Catanduva, com 16 municípios e São José do Rio Preto, com 30 municípios, no período de maio de 1991 a dezembro de 1994. Nas sub-regiões de Votuporanga, Fernandópolis, Jales e Santa Fé do Sul não foram encontradas amostras positivas contendo larvas de Ae. albopictus nas pesquisas para medida de Índices de Breteau. A Figura 1 registra o ano da primeira ocorrência de larvas do Ae. albopictus nos municípios da região, mostrando a evolução da sua colonização.
Na Tabela 2 são apresentadas as amostras com larvas de Ae. albopictus ou com ambas as espécies vetoras por ano em função do mês de ocorrência. Na Tabela 3 são apresentados os Índices de Breteau médios mensais separados por espécie.

Nas Tabelas 4 e 5 são apresentadas respectivamente a composição das amostras segundo localização e tipos de recipientes. Na Tabela 6 são apresentados os números médios de larvas de Ae. albopictus e Ae. aegypti por tipos de recipientes encontrados infestados no peridomicílio.

Tabela 1 - Nümero de amostras com laruas de Aedes albopictus encontradas has sub-regioes de Catanduva e São José do Rio Preto. São Paulo.

\begin{tabular}{|c|c|c|c|c|c|c|c|c|c|c|}
\hline \multirow{3}{*}{ Sub-regiôs } & \multicolumn{8}{|c|}{ Ano } & & \\
\hline & \multicolumn{2}{|c|}{1991} & \multicolumn{2}{|c|}{1992} & \multicolumn{2}{|c|}{1993} & \multicolumn{2}{|c|}{1994} & \multicolumn{2}{|c|}{ Total } \\
\hline & $n^{\mathrm{g}}$ & $\%$ & $\mathrm{n}^{\mathrm{o}}$ & $\%$ & $n^{0}$ & $\%$ & $\mathrm{n}^{\mathrm{Q}}$ & $\%$ & $\mathrm{n}^{\mathrm{g}}$ & $\%$ \\
\hline$\overline{\text { Catanduva }}$ & 4 & 3.10 & 7 & 5.50 & 11 & 8.60 & 106 & 82.80 & 128 & 100.0 \\
\hline São Josć do Rio Preto & 0 & 0,0 & 4 & 9.50 & 6 & 14.30 & 32 & 76.20 & 42 & 100,0 \\
\hline Total & 4 & 2.35 & 11 & 6,47 & 17 & 10.0 & 138 & 81.18 & 170 & 100,0 \\
\hline
\end{tabular}
1991 a 1994.

\begin{tabular}{|c|c|c|c|c|c|c|c|c|c|c|c|c|c|}
\hline \multirow[t]{2}{*}{ Ano } & \multicolumn{12}{|c|}{ Meses } & \multirow[t]{2}{*}{ Total } \\
\hline & Jan & Fev & Mar & $\mathrm{Abr}$ & Mai & Jun & Jul & Ago & Set & Out & $\mathrm{NOV}$ & Dez & \\
\hline 1991 & 0 & 0 & 0 & 0 & 4 & 0 & 0 & 0 & 0 & 0 & 0 & 0 & 4 \\
\hline 1992 & 1 & 0 & 2 & 2 & 5 & 0 & 0 & 1 & 0 & 0 & 0 & 0 & 11 \\
\hline 1993 & 1 & 3 & 0 & 4 & 4 & 0 & 0 & 0 & 0 & 0 & 0 & 5 & 17 \\
\hline 1994 & 22 & 59 & 35 & 0 & 3 & 5 & $\underline{2}$ & 3 & 0 & 0 & 0 & 9 & 138 \\
\hline Total & 24 & 62 & 37 & 6 & 16 & 5 & 2 & 4 & 0 & 0 & 0 & 14 & 170 \\
\hline$\%$ & 14,0 & 37.0 & 22,0 & 4.0 & 9.0 & 3,0 & 1.0 & 2.0 & 0.0 & 0.0 & 0.0 & 8,0 & 100.0 \\
\hline
\end{tabular}

Tabela 2 - Nümero de amostras com lantas de Aedes albopictus por ano e mês de ocomência. Regiäo de Säo José do Rio Preto. 1991 a 1994. 
Chiaravalloti Neto F, Costa AIP, Soares MRD. Scandlar SAS, Cardoso Jr RP. Descricão da colonização de Aedes albopictus (Diptera: Culicidae) na região de São José do Rio Preto, SP, 1991-1994. Revista da Sociedade Brasileira de Medicina Tropical 29:543-548, nov-dez 1996.

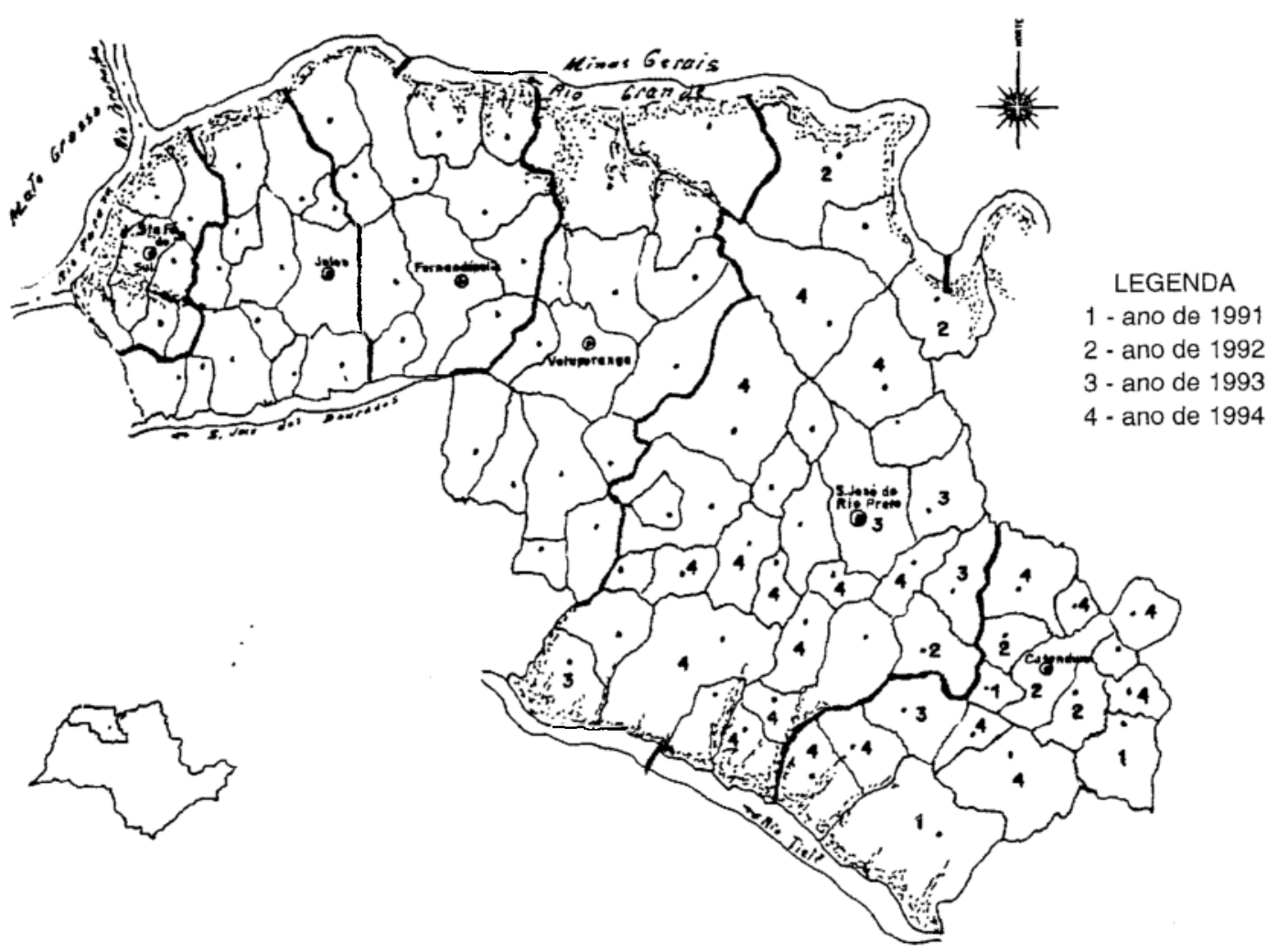

Figura 1 - Ano do primeiro encontro de larias de Aedes albopictus nos municípios da região de São José do Rio Preto, SP, 1991 a 1994.

Tabela 3 - Médias mensais de Indices de Breteau (IB) em municipios com ocorrência de lartas de Aedes albopictus. por espécie vetora e meses do ano. Regiáo de Sáo José do Rio Preto. São Paulo. 1994.

\begin{tabular}{lcccccccccccc}
\hline $\begin{array}{l}\text { IB por } \\
\text { espécie }\end{array}$ & Jan & Fer & Nar & Abr & Mai & Jun & Jul & Ago & Set & Out & Nov & Dez \\
Ae. albopictus & 0.61 & 0.90 & 0.71 & - & 0.40 & 0.47 & 0.45 & 0.37 & - & - & - & 0,51 \\
Ae. aeglpti & 7.19 & 7.54 & 4.48 & - & 6.11 & 1.65 & 2.02 & 0,00 & - & - & - & 1,00 \\
№ de medidas & 12 & 25 & 15 & 0 & 2 & 5 & 1 & 3 & 0 & 0 & 0 & 5 \\
\hline
\end{tabular}

Tabela 4 - Composiçào das amostras com larias de Aedes albopictus e/ou Aedes aegypti segundo localização. Regiäo de São José do Rio Preto. São Paulo. 1991 a 1994.

\begin{tabular}{|c|c|c|c|c|c|c|c|}
\hline \multirow{3}{*}{ Localizaçāo } & \multicolumn{6}{|c|}{ Composição* } & \multirow{3}{*}{$\begin{array}{r}\text { Total } \\
n^{\circ}\end{array}$} \\
\hline & \multicolumn{2}{|c|}{ Ae. albopictus } & \multicolumn{2}{|c|}{ ambas } & \multicolumn{2}{|c|}{ Ae. aegupti } & \\
\hline & $\mathrm{n}^{\mathrm{a}}$ & $\%$ & $n^{2}$ & $\%$ & $\mathrm{n}^{Q}$ & $\%$ & \\
\hline Intradomicílio & 1 & 1,43 & 5 & 6.49 & 522 & 17.51 & 528 \\
\hline Peridomicilio & 69 & 98.57 & 72 & 93.51 & 2460 & 82,49 & 2601 \\
\hline Total & 70 & 100.00 & 77 & 100.00 & 2982 & 100,00 & 3129 \\
\hline
\end{tabular}

"Qui-quadrado $=18.87: \mathrm{n}^{\circ}$ de graus de liberdade $=2: \mathrm{p}<0.0001$ 
Tabela 5 - Composiçāo das amostras com larvas de Aedes albopictus e/ou Aedes aegypti segundo tipos de recipientes encontrados no peridomicitio. Região de São José do Rio Preto. Säo Paulo, 1997 a 1994.

\begin{tabular}{|c|c|c|c|c|c|}
\hline \multirow{3}{*}{$\begin{array}{c}\text { Tipos } \\
\text { de } \\
\text { Recipientes }\end{array}$} & \multicolumn{4}{|c|}{ Espécies encontradas } & \multirow{3}{*}{ Total } \\
\hline & Ae. albopictus & Ambas & Sub-total & Ae. aegupti & \\
\hline & [1] & [2] & [3] & [4] & \\
\hline pneus & 13 & 11 & 24 & 293 & 317 \\
\hline recipientes para armazenamemto de água & 5 & 2 & 7 & 198 & 205 \\
\hline vasos e pratos de plantas & 10 & 17 & 27 & 787 & 814 \\
\hline bebedouros de animais & 9 & 10 & 19 & 173 & 192 \\
\hline latas, potes. frascos, copos & 18 & 22 & 40 & 584 & 624 \\
\hline recipientes fixos & 0 & 1 & 1 & 47 & 48 \\
\hline outros tipos de recipientes & 14 & 9 & 23 & 370 & 393 \\
\hline Total & 69 & 72 & 141 & 2452 & 2593 \\
\hline
\end{tabular}

Para as colunas 1 e 2 : Qui-quadrado $=5.75 ; \mathrm{n}^{9}$ graus de liberdade $=6: p=0.4523$

Para as colunas 3 e 4: Qui-quadrado $=21.31 ; \mathrm{n}^{\mathrm{Q}}$ graus de liberdade $=6 ; \mathrm{p}<0,002$.

Tabela 6 - Nümero médio de larvas de Aedes albopictus e Aedes aegypti por composiçāo das amostras, segundo tipos de recipientes. no peridomicilio. Regiäo de São José do Rio Preto, Säo Paulo, 1991 a 1994.

\begin{tabular}{|c|c|c|c|c|c|c|}
\hline \multirow{2}{*}{$\begin{array}{c}\text { Tipos } \\
\text { de } \\
\text { recipientes }\end{array}$} & \multicolumn{3}{|c|}{ Ae albopictus } & \multicolumn{3}{|c|}{$A e \cdot \overline{a e g} p t i$} \\
\hline & $\begin{array}{l}\text { Amostras com } \\
\text { Ae albopictus }\end{array}$ & $\begin{array}{c}\text { Amostra com } \\
\text { ambas espécies }\end{array}$ & $\begin{array}{c}\text { Probabilidade } \\
\qquad P^{*}\end{array}$ & $\begin{array}{c}\text { Amostras com } \\
\text { Ae. aegypti }\end{array}$ & $\begin{array}{l}\text { Amostras com } \\
\text { ambas espécies }\end{array}$ & $\begin{array}{c}\text { probalidade } \\
\mathrm{p}^{*}\end{array}$ \\
\hline pneus & 5,77 & 1.45 & $<0.05$ & 7.27 & 7,40 & $>0.05$ \\
\hline recipicntes para armazenamemto de água & 4.20 & 1,00 & $<0.05$ & 9.50 & 7,15 & $>0,05$ \\
\hline vasos e pratos de plantas & 3.60 & 4.00 & $>0,05$ & 8.35 & 7.22 & $>0.05$ \\
\hline bebedouros de animais & 3.67 & 1.80 & $<0.05$ & 7.70 & 7,66 & $>0,05$ \\
\hline latas, potes, frascos, copos & 5.28 & 2,09 & $<0.05$ & 7,68 & 8,22 & $>0,05$ \\
\hline outros tipos de recipientes & 5.86 & 2,11 & $<0.05$ & 5,56 & 6,75 & $>0,05$ \\
\hline Total & 4,96 & 2.38 & $<0.05$ & 7.61 & 7.43 & $>0.05$ \\
\hline
\end{tabular}

"Valores de "p" para o teste "I de Student" na comparação entre as médias (alfa $=0.05$ ).

\section{DISCUSSÃO}

Desde a primeira detecção de larvas de Aedes albopictus na região, as ocorrências foram muito pouco freqüentes até 1993. Já em 1994, houve um grande incremento, registrando-se $81 \%$ das ocorrências até então (138 a mostras em números absolutos), evidenciando o início de uma efetiva colonização. Esta seguiu a mesma direção assumida para o restante do Estado de São Paulo, de leste para $o_{\text {oste }}^{2}$, sendo primeiramente colonizados os municípios pertencentes à sub-região de Catanduva e, posteriormente, os da sub-região de São José do Rio Preto. Em 1994, apenas estas duas sub-regiões estavam colonizadas, com cerca de $76 \%$ das ocorrências na primeira sub-regiāo.

Em relação à sazonalidade, notou-se que $81 \%$ das ocorrências deram-se nos meses mais chuvosos: janeiro, fevereiro, março e dezembro, dados coincidentes com aqueles observados por Chiaravalloti Neto ${ }^{5}$ em relação ao $A e$. aegypti na região de São José do Rio Preto.

Os Índices de Breteau médios por espécie mostraram coerência com a recente colonização da região pelo Ae. albopictus, apresentando sempre valores bem inferiores para esta espécie em relação ao $A e$ aegypti, e o mesmo comportamento sazonal discutido acima.

Foi verificada a existência de uma associação estatisticamente significante entre a composição e a localização das amostras. Aquelas com presença de larvas de $A e$. albopictus ocorreram em maior proporção no peridomicílio quando comparadas com aquelas com a presença apenas de $A e$ aegypti, comportamento semelhante ao apontado por Forattini".

Com relação à ocupação de recipientes, as amostras com larvas de Ae. albopictus foram mais encontradas em latas, potes, frascos e copos, enquanto que as ocupadas somente por Ae. aegypti foram mais encontradas em vasos $e$ pratos de plantas. O teste do Qui-quadrado, comparando amostras com larvas de $A e$. albopictus acompanhadas ou não de larvas de Ae. aegupti com amostras somente de larvas de Ae. aegypti mostrou associação estatisticamente significante entre a composição das amostras e a ocupação dos recipientes. 
Cbiaralalloti Neto F, Costa AIP, Soares MRD, Scandlar SAS, Cardoso Jr RP. Descrição da colonização de Aedes albopictus (Diptera: Culicidae) na região de São José do Rio Preto, SP, 1991-1994. Rertsta da Sociedade Brasileira de Medicina Tropical 29:543-548, not-dez 1996.

O Ae. albopictus, quando encontrado isoladamente, apresentou um número médio de larvas sempre superior aquele encontrado quando em associação com Ae. aegvpti, sendo a diferença estatisticamente significante. $O$ mesmo aconteceu quando a comparação deuse por tipos de recipientes, com exceção de vasos e pratos de plantas.

A diferença entre os números médios de larvas de $A e$ aegypti encontradas em recipientes infestados apenas por Ae aegypti e por ambas espécies foi bastante reduzida e sem significância estatística, o mesmo ocorrendo quando a comparação se deu por tipos de recipientes, mostrando que o número médio de larvas de $A e$ aegypti não dependeu da presença do $A e$. albopictus.

Situaçôes distintas têm sido descritas quanto à interação entre as duas espécies. Em grandes cidades do Sudeste Asiático o Ae. aegypti temse expandido enquanto que a abundância do Ae. albopictus tem declinado em muitas dessas localidades. Nos EUA, a situação oposta tem ocorrido ${ }^{10}$. Segundo Service ${ }^{14}$, não há evidências, até o momento, de competição entre essas espécies.

Hawley $^{8}$ levanta a hipótese de que Ae. aegypti e Ae. albopictus no Sudeste Asiático ocupam nichos ecológicos distintos, sendo que o primeiro tem-se expandido por toda a região pela colonização de um nicho que só ocasionalmente é ocupado pelo Ae. albopictus (espécie natural da regiâo).

Conclui-se que a colonização da região pelo Ae. albopictus ainda é bastante reduzida, mas apresentou algumas diferenças com relação ao $A e$ aegypti: esteve presente em maior proporção no peridomicílio e ocupou recipientes em proporçôes diferentes. Apesar disso, não é possível afirmar até o momento que estejam ocupando nichos ecológicos distintos. O número médio de larvas de Ae. albopictus sofreu influência da presença de larvas da outra espécie, enquanto o contrário não ocorreu. Apresentou comportamento sazonal semelhante ao do $A e$ aegypti e avançou no sentido leste para oeste.

\section{SUMMARY}

This study aims to describe and analyze the colonization of Aedes albopictus whose presence was detected in 1991 in the area of São José do Rio Preto already colonized by the Aedes aegypti. Year and month of occurrence, connties, composition and location of larial samples, kinds of containers, azerage number of lamae an Breteau Index bave been analyzed from the information obtained in measurements of larial density by Superintendência de Controle de Endemias (SUCEN). The presence of Aedes albopictus was ascertained in 34 touns up to December 1994. The colonization of the area by the mosquito is still reduced showing some differences in relation to Aedes aegypti such as greater ratio outdoors, occupying container in different proportions. The average number of Aedes albopictus larrae has had influence of larae of another species. It has shoued a seasonal bebavior similar to Aedes aegypti and it bas mozed from east to west direction.

Key-uords: Aedes. Aedes albopictus. Colonization. Ecology.

\section{AGRADECIMENTOS}

Às funcionárias Beatriz A. C. Belini, Neuza F. A. Santana e Perpétua M. Magalhães, pela colaboração no levantamento de dados e ao funcionário Cláudio Martinez pela confecção da ilustração.

\section{REFERÊNCIAS BIBLIOGRAFICAS}

1. Alves MCP, Gurgel SM, Almeida MCRR. Plano amostral para cálculo de densidade larvária de Aedes aegypti e Aedes albopictus no Estado de São Paulo, Brasil. Revista de Saúde Pública 25:251256,1991 .

2. Burali GM, Marques GRAM, Gerola $J_{r} \mathrm{O}$. Distribuição geográfica de Aedes aegypti e Aedes albopictus no Estado de São Paulo. Revista da Sociedade Brasileira de Medicina Tropical, 24(supl. II): 182, 1991.

3. Brito M, Marques GRAM, Marques CCA, Tubaki RM. Primeiro encontro de Aedes (Stegomyia) albopictus (Skuse) no Estado de São Paulo, Brasil. Revista de Saúde Pública 20: 489,1986.

4. Chan KL. Singapore's dengue hemorragic fever control programe: a case study on the succesful control of Aedes aegypti and Aedes albopictus using mainly environmental as part of integrated vector control. Ministry of Health of Singapore, Singapore [mimeografado], 1985.

5. Chiaravalloti Neto F. Aedes aegypti na região de São José do Rio Preto, Estado de São Paulo. Tese de mestrado, Faculdade de Saúde Pública da Universidade de São Paulo, São Paulo, SP, 1993. 
Chiaravalloti Neto F, Costa AIP, Soares MRD, Scandlar SAS, Cardoso Jr RP. Descrição da colonização de Aedes albopictus (Diptera: Culicidae) na região de Sâo José do Rio Preto, SP, 1991-1994. Revista da Sociedade Brasileira de Medicina Tropical 29:543-548, noi-dez 1996.

6. Forattini OP. Identificação de Aedes (Stegomyia) albopictus (Skuse) no Brasil. Revista de Saúde Pública 20: 244-245,1986.

7. Guedes JS, Guedes MLS. Bioestatística para profissionais de saúde. Ao Livro Técnico, São Paulo, 1988.

8. Hawley WA. The biology of Aedes albopictus. Journal of American Mosquito Control Association 4:2-39, 1988.

9. Nobre A, Antezana D, Tauil PL. Febre amarela e dengue no Brasil: epidemiologia e controle. Revista da Sociedade Brasileira de Medicina Tropical 27(supl m):59-65,1994.

10. Rai KS. Aedes albopictus in the Americas. Annual Review of Entomology 36: 459-484, 1991.

11. Secretaria de Estado da Saúde. Vigilância epidemiológica do dengue. São Paulo [mimeo], 1987.
12. Secretaria de Estado da Saúde. Superintendência de Controle de Endemias. Plano de Emergência para o controle dos vetores do dengue e da febre amarela no verão de 1991/1992. São Paulo [mimeografado], 1991

13. Secretaria de Estado da Saúde. Superintendência de Controle de Endemias. Serviço Regional de São José do Rio Preto. Relatório de infestação pelo Aedes albopictus da região de São José do Rio Preto, São Paulo [mimeografado], 1992.

14. Service MW. Importance of ecologycal in Aedes aegypti control. Southeast Asean Journal of Tropical Medicine and Public Health 23: 681-689, 1992. 